Dhaka Univ. J. Biol. Sci. 31(1): 169-176, 2022 (January) ～DOI: https://doi.org/10.3329/dujbs.v31i1.57925

\title{
CHANGES IN PHOTOSYNTHETIC PIGMENT OF WHEAT ( TRITICUM AESTIVUM L. VAR. BARI GOM-25) AS AFFECTED BY NAA AND N-FERTILIZER
}

\author{
AMM Golam Adam ${ }^{* *}$, Nargis Jahan and Parveen Rashid \\ Department of Botany, University of Dhaka, Dhaka-1000 Bangladesh
}

Key words: Photosynthetic pigment, wheat, NAA, N-fertilizer

\begin{abstract}
A field experiment was conducted to investigate the changes in photosynthetic pigment of BARI Gom-25 as affected by foliar spray of NAA and $\mathrm{N}$-fertilizers. The experiment consisted of seven different concentrations of NAA $\left(\mathrm{NAA}_{0}=0, \mathrm{NAA}_{1}=10, \mathrm{NAA}_{2}=20, \mathrm{NAA}_{3}=30, \mathrm{NAA}_{4}=40, \mathrm{NAA}_{5}=50\right.$ and $\mathrm{NAA}_{6}=$ 60 ppm) and five N-levels $\left(F_{0}=0, F_{1}=25, F_{2}=50, F_{3}=75\right.$ and $F_{4}=100 \%$ of the recommended dose) and their 35 combination treatments. Results revealed that foliar application of 40 ppm NAA (NAA4) produced higher chlorophyll a content at all stages (tillering, flowering and grain filling) and were significantly higher than control at tilling and flowering stages. Chlorophyll $b$ and carotenoid contents did not show any general trend to NAA at any stage except at tilling stage. Application of $50 \%$ of the recommended $\mathrm{N}$-fertilizer $\left(\mathrm{F}_{2}\right)$ produced significantly maximum photosynthetic pigment contents at tilling stage whereas, at flowering stage the same results were obtained from $100 \%$ of the recommended $\mathrm{N}$-fertilizers $\left(\mathrm{F}_{4}\right)$ but with few exceptions. At grain filling stage, photosynthetic pigment did not show any general trend to N-fertilizer application. Findings on combined effects indicated that 20 and 30 ppm NAA in combination with $50 \% \mathrm{~N}$-fertilizer $\left(\mathrm{NAA}_{2} \mathrm{~F}_{2}\right.$ and $\left.\mathrm{NAA}_{3} \mathrm{~F}_{2}\right)$ had produced significantly maximum chlorophyll a content at tilling stage. However, at flowering stage the highest chlorophyll a content was noted from $\mathrm{NAA}_{2} \mathrm{~F}_{4}$ treatment (20 ppm NAA in combination with 100\% N-fertilizer). Findings also revealed that combined application of NAA and N-fertilizer had not resulted any general trend on chlorophyll $b$ and carotenoid contents in most of the cases where the maximum value in photosynthetic pigment was found depending on the concentrations of NAA, fertilizer dose and plant growth stages.
\end{abstract}

\section{Introduction}

Fertilization has a strong effect on productivity, nutritional quality management and the regulation of harvest formation processes ${ }^{(1)}$. Nitrogen $(\mathrm{N})$ fertilizers plays key role in agriculture and stimulating a lot of essential processes in plants. It is an important constituent of chlorophyll molecules which enables the plant to transfer energy from

*Author for correspondence: <adamammg@bot.jnu.ac.bd>. ${ }^{1}$ Department of Botany, Jagannath University, Dhaka-1100, Bangladesh. 
sunlight by photosynthesis. But excessive use of fertilizer is proven to cause a number of environmental and ecological problems within and outside of farmlands viz. air pollution, soil acidification and degradation, water eutrophication, crop yield reduction etc. (2-3). In addition, the amount of nitrogen applied to plants must be care-fully managed to ensure that $\mathrm{N}$ will be available throughout the growing season and the vegetative and reproductive development will be not restricted ${ }^{(4)}$. However, reports regarding the effects of $\mathrm{N}$ fertilizer on growth, yield, grain quality and nitrogen use efficiency are available from all over the world (5-6) but are lacking in relation to pigment contents(7).

On the other hand, the use of plant growth regulators especially Naphthalene acetic acid (NAA) has played dramatic role in reducing the demand of $\mathrm{N}$ fertilizers and producing improved results in wheat ${ }^{(8-9)}$. Previous investigations have also shown that application of optimum concentrations of NAA produced better stimulations in various cereal crops including wheat ${ }^{(10-11)}$. Thus, an attempt was taken to investigate the changes in photosynthetic pigment of wheat var. BARI Gom-25 as affected by NAA in combination with various N-levels.

\section{Materials and Methods}

A field experiment was conducted at the botanical garden of the Department of Botany, University of Dhaka. The experiment was laid out in randomized block design with four replications. The total area of the experimental field was $98.56 \mathrm{~m}^{2}(17.60 \mathrm{~m} \times$ $5.60 \mathrm{~m}$ ) and was prepared conventionally. Nitrogen, phosphorus and potassium content of experimental soil were determined by Micro-Kjeldahl's method(12), ascorbic acid blue colour method(13) and flame photometer(14), respectively. The initial properties of soil revealed low amount of nitrogen, very high amount of phosphorus and very low amount of potassium. Chemical fertilizers and cow-dung (4 ton/ha) were applied at doses recommended by Fertilizer Recommendation Guide 2012(15). Two-thirds of urea and full of the other fertilizers were applied as basal dose during final land preparation. The remaining urea was applied immediately after the first irrigation (18 days). A modern high yielding variety of wheat var. BARI Gom-25 was selected for this experiment. Seeds were collected from BARI, Joydebpur, Gazipur and were sterilized with $0.5 \%$ calcium hypochlorite before sowing. Seeds were sown on November 15, in lines $20 \mathrm{~cm}$ apart maintaining plant to plant distance of $10 \mathrm{~cm}$. Cultural practices viz., thinning, irrigation, weeding etc. were done following Hand Book of Agricultural Technology $y^{(16)}$.

The trial consisted of seven different treatments of NAA viz. NAA 0 foliar spray of distilled water only and $\mathrm{NAA}_{1}, \mathrm{NAA}_{2}, \mathrm{NAA}_{3}, \mathrm{NAA}_{4}, \mathrm{NAA}_{5}$ and $\mathrm{NAA}_{6}$ represent foliar spray of 10, 20, 30, 40,50 and 60 ppm NAA, respectively. There were also five levels of $\mathrm{N}$-fertilizers treatments viz. $\mathrm{F}_{0}=$ without application of urea and $\mathrm{F}_{1}, \mathrm{~F}_{2}, \mathrm{~F}_{3}, \mathrm{~F}_{4}=25,50,75$ and $100 \%$ of the recommended dose of urea. Considering seven foliar NAA and five levels of N-levels, this experiment comprised of 35 combination treatments. Foliar spray 
was done at the age of 30 days. Photosynthetic pigment viz. Chlorophyll a (Chl. a), Chlorophyll b (Chl. b) and total carotenoid contents of leaves were determined at tillering, flowering and grain filling stages. Fresh leaves were collected randomly from eight plants ( 2 from each replication) for each treatment at all stages. The analysis of pigments was determined spectrophotometrically. The amount of Chl. a and b were determined by using specific absorption co-efficient of McKinney ${ }^{(17)}$ and the formulae of Maclachalan and Zalik ${ }^{(18)}$. The amount of carotenoid were determined by the equation of von Wettstein ${ }^{(19)}$. Data were analyzed statistically and treatment means were compared by LSD test at $5 \%$ level of significance ${ }^{(20)}$.

\section{Results and Discussion}

Application of NAA and N-fertilizer as well as their combined effect had significant influences upon photosynthetic pigment contents of leaves at different stages but with few exceptions.

Photosynthetic pigment contents of leaves were positively influenced by NAA application at tillering stage except chl. $\mathrm{b}$ content due to $\mathrm{NAA}_{1}, \mathrm{NAA}_{2}$ and $\mathrm{NAA}_{3}$ treatments (Table 1). At this stage, all treatments resulted significantly higher Chl. a content except due to NAA treatment and the maximum value $(0.15 \mathrm{mg} / \mathrm{g})$ was recorded from $\mathrm{NAA}_{4}$ treatment. The range of increases were 12.50 to $175 \%$ whereas, the only decrease due to $\mathrm{NAA}_{1}$ was $50 \%$. However, application of NAA treatments had no significant effect on Chl. b content. Significantly higher amount of carotenoid content of leaves were recorded from all NAA treatments where, the highest increase due to NAA6 treatment was $66.42 \%$ followed by $\mathrm{NAA}_{4}$ treatment (63.75\%). At flowering stage, significantly higher Chl. a content of leaves was obtained from $\mathrm{NAA}_{4}$ and $\mathrm{NAA}_{6}$ treatments where the maximum increase due to NAA4 was $25 \%$ over the control. At this stage, Chl. $\mathrm{b}$ and carotenoid content obtained from NAA $\mathrm{NA}_{5}$ treatment although higher than control but statistically identical to each other. Application of $\mathrm{NAA}_{4}$ treatment although resulted higher Chl. a and carotenoid content at grain filling stage but statistically not different from control. At this stage significantly maximum carotenoid content was obtained from NAA4 treatment $(6.61 \mathrm{mg} / \mathrm{g})$.

Similarly, Singh and Gill (1985)(21) reported the stimulating effects of NAA on chlorophyll contents of leaves in wheat and barley. Depending on the concentrations of NAA both increase and decrease in photosynthetic pigment were obtained in maize ${ }^{(22)}$. Results of this experiment are also in accord with the findings of Jahan and Adam in two varieties of rice ${ }^{(23)}$.

Results presented in Table 1 showed that application of various doses of $\mathrm{N}$-fertilizers had significant responses on pigment contents of leaves at different stages. At the tillering stage, all the photosynthetic pigment contents were recorded maximum following $\mathrm{F}_{2}$ treatment whereas, minimum due to $\mathrm{F}_{1}$. Treatment $\mathrm{F}_{2}$ resulting maximum 
Chl. a and carotenoid were significantly different from the rest of the treatments. In case of $\mathrm{Chl} . \mathrm{b}$ content although the maximum and minimum were also recorded from $\mathrm{F}_{2}$ and $F_{1}$ treatments, respectively, where both are significantly different from each other but statistically similar to control.

Table 1. Effects of NAA and N-fertilizer on photosynthetic pigment (mg/g) of leaves of BARI Gom-25 at three different stages.

\begin{tabular}{|c|c|c|c|c|c|c|c|c|c|}
\hline \multirow[b]{2}{*}{ Treatments } & \multicolumn{3}{|c|}{ Tillering stage } & \multicolumn{3}{|c|}{ Flowering stage } & \multicolumn{3}{|c|}{ Grain filling stage } \\
\hline & Chl. a & Chl. b & $\begin{array}{l}\text { Caro- } \\
\text { tenoids }\end{array}$ & Chl. a & Chl. b & $\begin{array}{l}\text { Caro- } \\
\text { tenoids }\end{array}$ & Chl. a & Chl. b & $\begin{array}{l}\text { Caro- } \\
\text { tenoids }\end{array}$ \\
\hline \multicolumn{10}{|l|}{ NAA } \\
\hline $\mathrm{NAA}_{0}$ & $0.08 \mathrm{c}$ & $0.09 \mathrm{ab}$ & $4.11 \mathrm{f}$ & $0.20 \mathrm{~b}$ & $0.07 \mathrm{ab}$ & $6.70 \mathrm{a}$ & $0.19 \mathrm{ab}$ & $0.07 \mathrm{a}$ & $6.05 \mathrm{~b}$ \\
\hline $\mathrm{NAA}_{1}$ & $0.03 \mathrm{~d}$ & $0.06 \mathrm{~b}$ & $4.18 \mathrm{e}$ & $0.19 \mathrm{~b}$ & $0.06 \mathrm{bc}$ & $4.76 \mathrm{c}$ & $0.18 \mathrm{bc}$ & $0.06 \mathrm{a}$ & $5.81 \mathrm{~b}$ \\
\hline $\mathrm{NAA}_{2}$ & $0.13 \mathrm{ab}$ & $0.07 \mathrm{~b}$ & $5.88 \mathrm{c}$ & $0.19 \mathrm{~b}$ & $0.05 c$ & $5.52 \mathrm{~b}$ & $0.16 \mathrm{~d}$ & $0.07 \mathrm{a}$ & $5.22 \mathrm{c}$ \\
\hline $\mathrm{NAA}_{3}$ & $0.11 \mathrm{~b}$ & $0.07 \mathrm{~b}$ & $5.04 \mathrm{~d}$ & $0.20 \mathrm{~b}$ & $0.06 \mathrm{bc}$ & $6.36 \mathrm{a}$ & $0.19 \mathrm{ab}$ & $0.07 \mathrm{a}$ & $5.96 \mathrm{~b}$ \\
\hline $\mathrm{NAA}_{4}$ & $0.15 \mathrm{a}$ & $0.09 \mathrm{ab}$ & $6.73 \mathrm{ab}$ & $0.25 \mathrm{a}$ & $0.06 \mathrm{bc}$ & $6.39 \mathrm{a}$ & $0.20 \mathrm{a}$ & $0.07 \mathrm{a}$ & $6.09 \mathrm{~b}$ \\
\hline $\mathrm{NAA}_{5}$ & $0.13 \mathrm{ab}$ & $0.11 \mathrm{ab}$ & $6.51 \mathrm{~b}$ & $0.20 \mathrm{~b}$ & $0.08 \mathrm{a}$ & $6.78 \mathrm{a}$ & $0.19 \mathrm{ab}$ & $0.07 \mathrm{a}$ & $6.61 \mathrm{a}$ \\
\hline $\mathrm{NAA}_{6}$ & $0.13 \mathrm{ab}$ & $0.13 \mathrm{a}$ & $6.84 \mathrm{a}$ & $0.24 \mathrm{a}$ & $0.06 \mathrm{bc}$ & $6.64 \mathrm{a}$ & $0.17 \mathrm{~cd}$ & $0.04 \mathrm{a}$ & $5.37 \mathrm{c}$ \\
\hline \multicolumn{10}{|l|}{ N-Fertilizer } \\
\hline $\mathrm{F}_{0}$ & $0.08 \mathrm{c}$ & $0.10 \mathrm{ab}$ & $4.55 \mathrm{~d}$ & $0.21 \mathrm{c}$ & $0.07 \mathrm{a}$ & $6.87 \mathrm{~b}$ & $0.15 \mathrm{c}$ & $0.08 \mathrm{ab}$ & $5.67 \mathrm{~b}$ \\
\hline $\mathrm{F}_{1}$ & $0.04 \mathrm{~d}$ & $0.05 \mathrm{~b}$ & $4.47 \mathrm{~d}$ & $0.02 \mathrm{~d}$ & $0.04 \mathrm{~b}$ & $3.37 \mathrm{c}$ & $0.13 \mathrm{~d}$ & $0.09 \mathrm{a}$ & $6.13 \mathrm{a}$ \\
\hline $\mathrm{F}_{2}$ & $0.22 \mathrm{a}$ & $0.12 \mathrm{a}$ & $7.79 \mathrm{a}$ & $0.25 b$ & $0.07 \mathrm{a}$ & $6.72 \mathrm{~b}$ & $0.20 \mathrm{~b}$ & $0.05 \mathrm{~b}$ & $5.79 \mathrm{~b}$ \\
\hline $\mathrm{F}_{3}$ & $0.12 \mathrm{~b}$ & $0.10 \mathrm{ab}$ & $5.92 \mathrm{~b}$ & $0.24 b$ & $0.07 \mathrm{a}$ & $6.41 \mathrm{~b}$ & $0.22 \mathrm{a}$ & $0.06 \mathrm{ab}$ & $5.96 \mathrm{ab}$ \\
\hline $\mathrm{F}_{4}$ & $0.09 \mathrm{c}$ & $0.08 \mathrm{ab}$ & $5.32 \mathrm{c}$ & $0.33 \mathrm{a}$ & $0.07 \mathrm{a}$ & $7.44 \mathrm{a}$ & $0.20 \mathrm{~b}$ & $0.05 \mathrm{~b}$ & $5.65 \mathrm{~b}$ \\
\hline CV (\%) & 10.21 & 9.57 & 14.05 & 18.03 & 17.96 & 12.50 & 19.63 & 14.44 & 9.32 \\
\hline $\mathrm{LSD}(0.05)$ & 0.02 & 0.05 & 0.32 & 0.01 & 0.01 & 0.48 & 0.01 & 0.03 & 0.33 \\
\hline
\end{tabular}

Mean in a vertical column followed by same letter or without letter do not differ significantly at $5 \%$ level.

At flowering stage, significantly higher Chl. a (57.14\%) and carotenoid (8.30\%) were obtained from $\mathrm{F}_{4}$ treatment whereas, $\mathrm{Chl}$. b content was affected with similar statistical values. Results revealed that $F_{1}$ treatment produced significantly lowest photosynthetic pigment at this stage.

At grain filling stage, significantly higher amount of $\mathrm{Chl}$. a was recorded from $\mathrm{F}_{2}, \mathrm{~F}_{3}$ and $F_{4}$ treatments and increased by 33.33, 46.67 and $33.33 \%$, respectively. Different doses of $\mathrm{N}$-fertilizer resulted higher carotenoid content of leaves where, $\mathrm{F}_{1}$ treatment produced $8.11 \%$ higher value over the control. However, the maximum $\mathrm{Chl}$. $\mathrm{b}$ was obtained from $\mathrm{F}_{1}$ and the minimum from $\mathrm{F}_{2}$ and both these are significantly different from each other but statistically at par with the control.

Present findings indicated that photosynthetic pigment are very sensitive to nitrogen levels and $50 \%$ of the recommended dose $\left(\mathrm{F}_{2}\right)$ produced maximum photosynthetic pigments at tillering stage whereas at flowering stage the maximum was noted from 
$100 \%$ of the recommended dose $\left(\mathrm{F}_{4}\right)$ treatment. But no general trend was found in photosynthetic pigment contents at grain filling stage. Although reports regarding the effect of N-levels on photosynthetic pigments are scanty. But, findings of Tranavičienè et al.(7) reveled that total photosynthetic pigment contents increased with plant age and were higher at higher fertilization rates.

Pigment contents of leaves were both significantly and non-significantly influenced by NAA at varying N-levels (Table 2). At tillering stage, Chl. a content of leaves was obtained maximum $(0.38 \mathrm{mg} / \mathrm{g})$ following both $\mathrm{NAA}_{2} \mathrm{~F}_{2}$ and $\mathrm{NAA}_{3} \mathrm{~F}_{2}$ treatments and were significantly different from rest of the treatments. The least amount of Chl. a $(0.01 \mathrm{mg} / \mathrm{g})$ was recorded from the leaves of $\mathrm{NAA}_{0} \mathrm{~F}_{1}$ and $\mathrm{NAA}_{3} \mathrm{~F}_{4}$ treatments and were significantly different from control. Combined treatments had retarding effect on Chl. $\mathrm{b}$ content of leaves although $\mathrm{NAA}_{6} \mathrm{~F}_{2}$ and $\mathrm{NAA}_{6} \mathrm{~F}_{3}$ treatments produced higher values but statistical identical to control. At this stage, carotenoid content of leaves were significantly higher following all treatments where the maximum increase due to $\mathrm{NAA}_{2} \mathrm{~F}_{2}$ treatment was $383.08 \%$ followed by $\mathrm{NAA}_{4} \mathrm{~F}_{3}$ treatment $(380 \%)$.

Table 2. Combined effects of NAA and N-fertilizer on photosynthetic pigment (mg/g) of leaves of BARI Gom-25 at three different stages.

\begin{tabular}{|c|c|c|c|c|c|c|c|c|c|}
\hline \multirow{2}{*}{$\begin{array}{l}\text { Treatments } \\
(\mathrm{NAA} \times \mathrm{F})\end{array}$} & \multicolumn{3}{|c|}{ Tillering stage } & \multicolumn{3}{|c|}{ Flowering stage } & \multicolumn{3}{|c|}{ Grain filling stage } \\
\hline & Chl. a & Chl. b & $\begin{array}{l}\text { Caro- } \\
\text { tenoid }\end{array}$ & Chl. a & Chl. b & $\begin{array}{l}\text { Caro- } \\
\text { tenoid }\end{array}$ & Chl. a & Chl. b & $\begin{array}{l}\text { Caro- } \\
\text { tenoid }\end{array}$ \\
\hline $\mathrm{NAA}_{0} \mathrm{~F}_{0}$ & $0.09 \mathrm{e}$ & $0.16 \mathrm{a}-\mathrm{d}$ & $1.95 \mathrm{p}$ & $0.22 \mathrm{ij}$ & $0.08 \mathrm{bc}$ & $7.95 \mathrm{c}-\mathrm{e}$ & $0.21 \mathrm{ef}$ & $0.12 \mathrm{a}$ & $7.30 \mathrm{bc}$ \\
\hline $\mathrm{NAA}_{1} \mathrm{~F}_{0}$ & $0.08 \mathrm{ef}$ & $0.11 \mathrm{~d}-\mathrm{g}$ & $4.40 \mathrm{jk}$ & $0.21 \mathrm{j}$ & $0.06 \mathrm{de}$ & $6.43 \mathrm{~lm}$ & 0.08 op & $0.11 \mathrm{a}$ & $4.83 \mathrm{n}$ \\
\hline $\mathrm{NAA}_{2} \mathrm{~F}_{0}$ & $0.18 \mathrm{~d}$ & $0.12 \mathrm{~d}-\mathrm{f}$ & $6.80 \mathrm{e}$ & 0.06 o & $0.02 \mathrm{hi}$ & $2.87 \mathrm{u}$ & $0.07 \mathrm{p}$ & $0.06 \mathrm{~cd}$ & $3.30 \mathrm{q}$ \\
\hline $\mathrm{NAA}_{3} \mathrm{~F}_{0}$ & $0.08 \mathrm{ef}$ & $0.12 \mathrm{~d}-\mathrm{f}$ & $5.50 \mathrm{~g}$ & $0.13 \mathrm{~lm}$ & $0.07 \mathrm{~cd}$ & 6.491 & $0.21 \mathrm{ef}$ & $0.11 \mathrm{a}$ & $7.57 \mathrm{~b}$ \\
\hline $\mathrm{NAA}_{4} \mathrm{~F}_{0}$ & $0.05 \mathrm{gh}$ & $0.11 \mathrm{~d}-\mathrm{g}$ & $5.95 \mathrm{f}$ & $0.35 \mathrm{~b}$ & $0.07 \mathrm{~cd}$ & $7.85 \mathrm{c}-\mathrm{f}$ & $0.13 \mathrm{~m}$ & $0.06 \mathrm{~cd}$ & $4.981-n$ \\
\hline $\mathrm{NAA}_{5} \mathrm{~F}_{0}$ & $0.02 \mathrm{ij}$ & $0.03 \mathrm{i}$ & $2.62 \mathrm{o}$ & 0.141 & $0.12 \mathrm{a}$ & $8.01 \mathrm{~b}-\mathrm{d}$ & $0.18 \mathrm{hi}$ & $0.06 \mathrm{~cd}$ & $6.85 \mathrm{~d}-\mathrm{f}$ \\
\hline $\mathrm{NAA}_{6} \mathrm{~F}_{0}$ & $0.02 \mathrm{ij}$ & $0.05 \mathrm{hi}$ & $4.60 \mathrm{ij}$ & $0.34 \mathrm{~b}$ & $0.08 \mathrm{bc}$ & $8.48 \mathrm{ab}$ & $0.13 \mathrm{~m}$ & $0.04 \mathrm{~cd}$ & $4.86 n$ \\
\hline $\mathrm{NAA}_{0} \mathrm{~F}_{1}$ & $0.01 \mathrm{j}$ & $0.03 \mathrm{i}$ & 2.76 no & $0.04 \mathrm{p}$ & $0.12 \mathrm{a}$ & $4.92 \mathrm{pq}$ & $0.14 \mathrm{~lm}$ & $0.07 \mathrm{bc}$ & $5.86 \mathrm{gh}$ \\
\hline $\mathrm{NAA}_{1} \mathrm{~F}_{1}$ & $0.02 \mathrm{ij}$ & $0.04 \mathrm{i}$ & $4.79 \mathrm{hi}$ & $0.02 \mathrm{q}$ & $0.05 \mathrm{ef}$ & $1.25 \mathrm{w}$ & 0.21 ef & $0.06 \mathrm{~cd}$ & $6.80 \mathrm{ef}$ \\
\hline $\mathrm{NAA}_{2} \mathrm{~F}_{1}$ & $0.05 \mathrm{gh}$ & $0.03 \mathrm{i}$ & $4.57 \mathrm{ij}$ & $0.01 \mathrm{q}$ & $0.01 \mathrm{i}$ & $4.78 \mathrm{q}$ & 0.09 no & $0.10 a b$ & $4.85 \mathrm{n}$ \\
\hline $\mathrm{NAA}_{3} \mathrm{~F}_{1}$ & $0.06 \mathrm{fg}$ & $0.03 \mathrm{i}$ & $4.68 \mathrm{ij}$ & $0.02 \mathrm{q}$ & $0.03 \mathrm{gh}$ & $4.24 \mathrm{r}$ & $0.10 \mathrm{n}$ & $0.11 \mathrm{a}$ & $6.62 \mathrm{f}$ \\
\hline $\mathrm{NAA}_{4} \mathrm{~F}_{1}$ & $0.03 \mathrm{~h}-\mathrm{j}$ & $0.07 \mathrm{f}-\mathrm{i}$ & $4.74 \mathrm{i}$ & $0.01 \mathrm{q}$ & $0.01 \mathrm{i}$ & $2.36 \mathrm{v}$ & $0.13 \mathrm{~m}$ & $0.11 \mathrm{a}$ & $5.37 \mathrm{i}-\mathrm{k}$ \\
\hline $\mathrm{NAA}_{5} \mathrm{~F}_{1}$ & $0.03 \mathrm{~h}-\mathrm{j}$ & $0.07 \mathrm{f}-\mathrm{i}$ & $5.07 \mathrm{~h}$ & $0.02 \mathrm{q}$ & $0.02 \mathrm{hi}$ & $3.40 \mathrm{~s}$ & $0.13 \mathrm{~m}$ & $0.13 \mathrm{a}$ & $8.09 \mathrm{a}$ \\
\hline $\mathrm{NAA}_{6} \mathrm{~F}_{1}$ & $0.09 \mathrm{e}$ & $0.07 \mathrm{f}-\mathrm{i}$ & $4.71 \mathrm{ij}$ & $0.02 \mathrm{q}$ & $0.02 \mathrm{hi}$ & $2.64 \mathrm{uv}$ & $0.15 \mathrm{kl}$ & $0.04 \mathrm{~cd}$ & $5.29 \mathrm{j}-1$ \\
\hline $\mathrm{NAA}_{0} \mathrm{~F}_{2}$ & $0.06 \mathrm{fg}$ & $0.07 \mathrm{f}-\mathrm{i}$ & $5.56 \mathrm{~g}$ & $0.29 \mathrm{ef}$ & $0.07 \mathrm{~cd}$ & $7.41 \mathrm{f}-\mathrm{i}$ & $0.14 \mathrm{~lm}$ & $0.04 \mathrm{~cd}$ & $4.42 \mathrm{o}$ \\
\hline $\mathrm{NAA}_{1} \mathrm{~F}_{2}$ & $0.02 \mathrm{ij}$ & $0.07 \mathrm{f}-\mathrm{i}$ & $4.18 \mathrm{kl}$ & $0.10 \mathrm{n}$ & $0.04 \mathrm{fg}$ & $3.36 \mathrm{~s}$ & $0.17 \mathrm{ij}$ & $0.04 \mathrm{~cd}$ & $5.21 \mathrm{j}-\mathrm{m}$ \\
\hline
\end{tabular}




\begin{tabular}{|c|c|c|c|c|c|c|c|c|c|}
\hline \multirow{2}{*}{$\begin{array}{l}\text { Treatments } \\
(\mathrm{NAA} \times \mathrm{F})\end{array}$} & \multicolumn{3}{|c|}{ Tillering stage } & \multicolumn{3}{|c|}{ Flowering stage } & \multicolumn{3}{|c|}{ Grain filling stage } \\
\hline & Chl. a & Chl. b & $\begin{array}{l}\text { Caro- } \\
\text { tenoid }\end{array}$ & Chl. a & Chl. b & $\begin{array}{l}\text { Caro- } \\
\text { tenoid }\end{array}$ & Chl. a & Chl. b & $\begin{array}{l}\text { Caro- } \\
\text { tenoid }\end{array}$ \\
\hline $\mathrm{NAA}_{2} \mathrm{~F}_{2}$ & $0.38 \mathrm{a}$ & $0.10 \mathrm{e}-\mathrm{h}$ & $9.42 \mathrm{a}$ & $0.19 \mathrm{k}$ & $0.06 \mathrm{de}$ & $5.51 \mathrm{no}$ & $0.20 \mathrm{fg}$ & $0.06 \mathrm{~cd}$ & $4.88 \mathrm{mn}$ \\
\hline $\mathrm{NAA}_{3} \mathrm{~F}_{2}$ & $0.38 \mathrm{a}$ & $0.11 \mathrm{~d}-\mathrm{g}$ & $9.35 \mathrm{a}$ & $0.30 \mathrm{de}$ & $0.08 \mathrm{bc}$ & $7.63 \mathrm{~d}-\mathrm{h}$ & $0.21 \mathrm{ef}$ & $0.04 \mathrm{~cd}$ & $5.11 \mathrm{k}-\mathrm{n}$ \\
\hline $\mathrm{NAA}_{4} \mathrm{~F}_{2}$ & $0.31 \mathrm{~b}$ & $0.11 \mathrm{~d}-\mathrm{g}$ & $8.83 \mathrm{~b}$ & $0.28 \mathrm{fg}$ & $0.08 \mathrm{bc}$ & $7.06 \mathrm{i}-\mathrm{k}$ & $0.24 \mathrm{bc}$ & $0.06 \mathrm{~cd}$ & $7.13 \mathrm{c}-\mathrm{e}$ \\
\hline $\mathrm{NAA}_{5} \mathrm{~F}_{2}$ & $0.17 \mathrm{~d}$ & $0.16 \mathrm{a}-\mathrm{d}$ & $8.42 \mathrm{c}$ & $0.30 \mathrm{de}$ & $0.09 \mathrm{~b}$ & $8.30 \mathrm{a}-\mathrm{c}$ & $0.25 \mathrm{~b}$ & $0.06 \mathrm{~cd}$ & $7.16 \mathrm{~cd}$ \\
\hline $\mathrm{NAA}_{6} \mathrm{~F}_{2}$ & $0.25 \mathrm{c}$ & $0.19 \mathrm{ab}$ & $8.79 \mathrm{~b}$ & $0.28 \mathrm{fg}$ & $0.09 \mathrm{~b}$ & $7.76 \mathrm{~d}-\mathrm{g}$ & $0.22 \mathrm{de}$ & $0.05 \mathrm{~cd}$ & $6.61 \mathrm{f}$ \\
\hline $\mathrm{NAA}_{0} \mathrm{~F}_{3}$ & $0.05 \mathrm{gh}$ & $0.07 \mathrm{f}-\mathrm{i}$ & $3.59 \mathrm{~m}$ & $0.12 \mathrm{~m}$ & $0.04 \mathrm{fg}$ & $5.31 \mathrm{op}$ & $0.24 \mathrm{bc}$ & $0.06 \mathrm{~cd}$ & $6.12 \mathrm{~g}$ \\
\hline $\mathrm{NAA}_{1} \mathrm{~F}_{3}$ & $0.04 \mathrm{~g}-\mathrm{i}$ & $0.06 \mathrm{~g}-\mathrm{i}$ & $3.47 \mathrm{~m}$ & $0.31 \mathrm{~cd}$ & $0.06 \mathrm{de}$ & $4.88 \mathrm{pq}$ & $0.21 \mathrm{ef}$ & $0.05 \mathrm{~cd}$ & $5.47 \mathrm{ij}$ \\
\hline $\mathrm{NAA}_{2} \mathrm{~F}_{3}$ & $0.04 \mathrm{~g}-\mathrm{i}$ & $0.06 \mathrm{~g}-\mathrm{i}$ & 4.001 & $0.23 \mathrm{i}$ & $0.06 \mathrm{de}$ & $5.95 \mathrm{mn}$ & $0.24 \mathrm{bc}$ & $0.06 \mathrm{~cd}$ & $6.17 \mathrm{~g}$ \\
\hline $\mathrm{NAA}_{3} \mathrm{~F}_{3}$ & $0.04 \mathrm{~g}-\mathrm{i}$ & $0.07 \mathrm{f}-\mathrm{i}$ & $2.99 \mathrm{n}$ & $0.26 \mathrm{~h}$ & $0.07 \mathrm{~cd}$ & $6.82 \mathrm{j}-1$ & $0.24 \mathrm{bc}$ & $0.05 \mathrm{~cd}$ & $6.15 \mathrm{~g}$ \\
\hline $\mathrm{NAA}_{4} \mathrm{~F}_{3}$ & $0.31 \mathrm{~b}$ & $0.14 \mathrm{~b}-\mathrm{e}$ & $9.36 \mathrm{a}$ & $0.31 \mathrm{~cd}$ & $0.07 \mathrm{~cd}$ & 7.47 e-i & $0.27 \mathrm{a}$ & $0.07 b c$ & $6.78 \mathrm{f}$ \\
\hline $\mathrm{NAA}_{5} \mathrm{~F}_{3}$ & $0.27 \mathrm{c}$ & $0.11 \mathrm{~d}-\mathrm{g}$ & $8.74 \mathrm{bc}$ & $0.22 \mathrm{ij}$ & $0.09 \mathrm{~b}$ & $7.17 \mathrm{~h}-\mathrm{j}$ & $0.16 \mathrm{jk}$ & $0.04 \mathrm{~cd}$ & $4.88 \mathrm{mn}$ \\
\hline $\mathrm{NAA}_{6} \mathrm{~F}_{3}$ & $0.08 \mathrm{ef}$ & $0.20 \mathrm{a}$ & $9.29 \mathrm{a}$ & $0.27 \mathrm{gh}$ & $0.07 \mathrm{~cd}$ & $7.31 \mathrm{~g}-\mathrm{i}$ & $0.21 \mathrm{ef}$ & $0.06 \mathrm{~cd}$ & $6.12 \mathrm{~g}$ \\
\hline $\mathrm{NAA}_{0} \mathrm{~F}_{4}$ & $0.18 \mathrm{~d}$ & $0.11 \mathrm{~d}-\mathrm{e}$ & $6.66 \mathrm{e}$ & $0.30 \mathrm{de}$ & $0.03 \mathrm{gh}$ & $7.91 \mathrm{c}-\mathrm{e}$ & $0.21 \mathrm{ef}$ & $0.05 \mathrm{~cd}$ & $6.54 \mathrm{f}$ \\
\hline $\mathrm{NAA}_{1} \mathrm{~F}_{4}$ & $0.02 \mathrm{ij}$ & $0.03 \mathrm{i}$ & 4.071 & $0.32 \mathrm{c}$ & $0.09 \mathrm{~b}$ & $7.89 \mathrm{c}-\mathrm{f}$ & $0.25 \mathrm{~b}$ & $0.05 \mathrm{~cd}$ & $6.76 \mathrm{f}$ \\
\hline $\mathrm{NAA}_{2} \mathrm{~F}_{4}$ & $0.02 \mathrm{ij}$ & $0.03 \mathrm{i}$ & $4.62 \mathrm{ij}$ & $0.45 \mathrm{a}$ & $0.11 \mathrm{a}$ & $8.52 \mathrm{a}$ & $0.19 \mathrm{gh}$ & $0.06 \mathrm{~cd}$ & $5.67 \mathrm{hi}$ \\
\hline $\mathrm{NAA}_{3} \mathrm{~F}_{4}$ & $0.01 \mathrm{j}$ & $0.02 \mathrm{i}$ & $2.66 \mathrm{o}$ & $0.30 \mathrm{de}$ & $0.07 \mathrm{~cd}$ & $6.60 \mathrm{kl}$ & $0.17 \mathrm{ij}$ & $0.05 \mathrm{~cd}$ & $4.34 \mathrm{o}$ \\
\hline $\mathrm{NAA}_{4} \mathrm{~F}_{4}$ & $0.03 \mathrm{~h}-\mathrm{j}$ & $0.02 \mathrm{i}$ & $4.76 \mathrm{hi}$ & $0.32 \mathrm{c}$ & $0.07 \mathrm{~cd}$ & $7.18 \mathrm{~h}-\mathrm{j}$ & $0.22 \mathrm{de}$ & $0.05 \mathrm{~cd}$ & $6.18 \mathrm{~g}$ \\
\hline $\mathrm{NAA}_{5} \mathrm{~F}_{4}$ & $0.17 \mathrm{~d}$ & $0.18 \mathrm{a}-\mathrm{c}$ & $7.69 \mathrm{~d}$ & $0.31 \mathrm{~cd}$ & $0.08 \mathrm{bc}$ & $7.02 \mathrm{i}-\mathrm{k}$ & $0.23 \mathrm{~cd}$ & $0.07 \mathrm{bc}$ & $6.09 \mathrm{~g}$ \\
\hline $\mathrm{NAA}_{6} \mathrm{~F}_{4}$ & $0.19 \mathrm{~d}$ & $0.13 c-e$ & $6.81 \mathrm{e}$ & $0.30 \mathrm{de}$ & $0.06 \mathrm{de}$ & $7.00 \mathrm{i}-\mathrm{k}$ & 0.14 & $0.03 \mathrm{~d}$ & $3.95 \mathrm{p}$ \\
\hline CV (\%) & 10.21 & 9.57 & 14.05 & 18.03 & 17.96 & 12.50 & 19.63 & 14.44 & 9.32 \\
\hline LSD (0.05) & 0.02 & 0.05 & 0.32 & 0.01 & 0.01 & 0.48 & 0.01 & 0.03 & 0.33 \\
\hline
\end{tabular}

Mean in a vertical column followed by same letter or without letter do not differ significantly at $5 \%$ level.

At flowering stage, the maximum increase in Chl. a content due to $\mathrm{NAA}_{2} \mathrm{~F}_{4}$ treatment was $104.55 \%$ over the control followed by $59.09 \%\left(\mathrm{NAA}_{4} \mathrm{~F}_{0}\right)$ and $55.55 \%\left(\mathrm{NAA}_{6} \mathrm{~F}_{0}\right)$, respectively (Table 2). The maximum amount of Chl. b content of leaves was observed from two treatments, $\mathrm{NAA}_{5} \mathrm{~F}_{0}$ and $\mathrm{NAA}_{0} \mathrm{~F}_{1}$. Increases in $\mathrm{Chl}$. $\mathrm{b}$ content due to $\mathrm{NAA}_{5} \mathrm{~F}_{0}$, $\mathrm{NAA}_{0} \mathrm{~F}_{1}$ and $\mathrm{NAA}_{2} \mathrm{~F}_{4}$ treatments were $50.0,50.0$ and $37.50 \%$ over the control respectively. Significantly maximum carotenoid content of leaves was observed from $\mathrm{NAA}_{2} \mathrm{~F}_{4}$ treatment which was $7.17 \%$ higher over the control followed by $6.67 \%$ ( $\left.\mathrm{NAA}_{6} \mathrm{~F}_{0}\right), 4.40 \%$ $\left(\mathrm{NAA}_{5} \mathrm{~F}_{2}\right)$ and $0.75 \%\left(\mathrm{NAA}_{5} \mathrm{~F}_{0}\right)$ treatments respectively.

At grain filling stage, only a few combined treatments $\left(\mathrm{NAA}_{4} \mathrm{~F}_{2}, \mathrm{NAA}_{5} \mathrm{~F}_{2}, \mathrm{NAA}_{0} \mathrm{~F}_{3}\right.$, $\mathrm{NAA}_{2} \mathrm{~F}_{3}, \mathrm{NAA}_{3} \mathrm{~F}_{3}, \mathrm{NAA}_{4} \mathrm{~F}_{3}, \mathrm{NAA}_{1} \mathrm{~F}_{4}, \mathrm{NAA}_{5} \mathrm{~F}_{4}$ ) had significant beneficial effect on Chl. a 
content of leaves. But in case of Chl. b content of leaves it was affected almost negatively having an exception due to $\mathrm{NAA}_{5} \mathrm{~F}_{1}$ treatment. Significantly maximum $(8.09 \mathrm{mg} / \mathrm{g})$ carotenoid content of leaves at grain filling stage was due to $\mathrm{NAA}_{5} \mathrm{~F}_{1}$ treatment where it was $10.82 \%$ higher over the control. However, the highest decrease in carotenoid content was $54.79 \%$ due to $\mathrm{NAA}_{2} \mathrm{~F}_{0}$ treatment. Islam $2015^{(24)}$ obtained significantly higher $\mathrm{Chl}$. a and Chl. $b$ content of leaves of BARI Gom-26 following NAA in combination with various $\mathrm{N}$-levels at flowering and grain filling stages but with few exceptions. However, combination of NAA and N-fertilizer had not resulted any significant difference in carotenoid content of leaves. Application of NAA at varying N-level produced negative influences in pigment content of leaves at tassel initiation stage and both stimulatory and retarding effects at grain filling stage of maize ${ }^{(25)}$.

\section{Conclusion}

In plants, utilization of solar energy mainly depends on the relative amount and efficiency of the photosynthetic pigments. Chlorophyll content of leaf is an important factor for increasing or decreasing photosynthesis of any crop plant. It was evident that application of NAA, N-fertilizer and their combined treatments had both stimulatory and retarding effects on pigment content of leaves at different stages of BARI Gom-25. Findings also revealed that combined application of NAA and N-fertilizer had not produced any general trend on leaf pigments and the maximum value in photosynthetic pigment was obtained depending on the concentrations of NAA, fertilizer dose and plant growth stages.

\section{References}

1. Ewert F and B Honermeier 1999. Spikelet initiation of winter triticale and winter wheat in response to nitrogen fertilization. European J. Agron. 11(2): 107-113.

2. Vitousek PM, R Naylor, T Crews, MB David, LE Drinkwater, E Holland, PJ Johnes, J Katzenberger, LA Martinelli, PA Matson and G Nziguheba 2009. Nutrient imbalances inagricultural development. Science. 324: 1519-1520.

3. Sutton MA, O Oenema, JW Erisman, A Leip, H van Grinsven, and W Winiwarter 2011. Too much of a good thing. Nature: 472: 159-161.

4. Vidal I, L Longeri and JM Hetier 1999. Nitrogen uptake and chlorophyll meter measurements in spring wheat. Nutr. Cycl. Agroecosystems 555: 1-6.

5. Guarda G, S Padovan, G Delogu 2004. Grain yield, nitrogen-use efficiency and baking quality of old and modern Italian bread-wheat cultivars grown at different nitrogen levels. Eur. J. Agron. 21: 181-192.

6. Dandan L and Y Shi 2013. Effects of different nitrogen fertilizer on quality and yield in winter wheat. Adv. J. Food Sci. Technol. 5(5): 646-649.

7. Tranavičienė T, JB Šikšnianienė, AUrbonavičiūtè, I Vagusevičienė, G Samuolienė, P Duchovskis, A Sliesaravičius2007. Effects of nitrogen fertilizers on wheat photosynthetic pigment and carbohydrate contents. Biologija. 53(4): 80-84. 
8. Islam S and N Jahan 2016. Growth and yield responses of Triticum_aestivum L. var. BARI Gom-26 following application of Naphthalene acetic acid (NAA) at varying nitrogen levels. Bangladesh J. Bot. 45(2): 411-418.

9. Adam AMMG, N Jahan and P Rashid 2020. Effects of NAA and nitrogen fertilizer on NPK uptake and nitrogen use efficiency of BARI Gom-25 (Triticum_aestivum L). Bangladesh J. Bot. 49(3): 643-651.

10. Adam AMMG and N Jahan 2011. Effects of Naphthalene acetic acid (NAA) on yield attributes and yield of two varieties of rice (Oryza sativa L.). Bangladesh J. Bot. 40(1): 97-100.

11. Jahan N and AMMG Adam 2013. Growth and yield response of BARI Gom-26 (Triticum aestivum L.) to Naphthalene acetic acid. Dhaka Univ. J. Biol. Sci. 22(2): 119-125.

12. Marr IL and MS Cresser 1983. Environmental chemical analysis. University of California. International Textbook Co. pp. 258.

13. Murphy J and JP Riley 1962. A modified simple solution method for determination of phosphate in saturated water. Analytica. Chemica. Acta. 27: 31-36.

14. Jackson ML 1958. Soil Chemical Analysis. Prentice-Hall Inc., Englewood Cliffs, NJ, USA. pp. 498.

15. Fertilizer Recommendation Guide 2012. BARC, Farmgate, Dhaka-1215. pp. 274.

16. Chowdhury MAH and MS Hassan 2013. Handbook of Agricultural Technology. Bangladesh Agricultural Technology. BARC, Farmgate, Dhaka. pp. 230.

17. Mckinney G 1940. Criteria for purity of chlorophyll preparations. J. Biol. Chem. 132: 91-107.

18. Maclachalan S and S Zalik 1963. Plastid structure, chlorophyll concentration and free amino acid composition of a chlorophyll mutant of barley. Can. J. Bot. 41: 1053-1062.

19. von Wettstein D 1957. Chlorophyll-lethal under Submikroskopisoche Formechse der Plastiden. Expt. Cell Res. 12: 427-507.

20. Steel RGD, JH Torrie and DA Dickey 1997. Principles and Procedures of Statistics. McGraw Hill Book Co. Inc. New York. pp. 666.

21. Singh H and HS Gill 1985. Effect of foliar spray of NAA on the growth and yield of late sown wheat and barley. Indian J. Eco. 12: 267-272.

22. Akter R 2010. Effect of Naphthalene acetic acid (NAA) on growth, physiological and biochemical responses and yield attributes of maize (Zea_mays L. var. Pacific 283). MS Thesis, Department of Botany, University of Dhaka.

23. Jahan N and AMMG Adam 2014. Changes in biochemical components of rice following NAA application. J. Asiat. Soc. Bangladesh Sci. 40(2): 173- 178.

24. Islam S 2015. Effect of Naphthalene acetic acid (NAA) at varying nitrogen levels on wheat (Triticum aestivum L. var. BARI Gom-26). MS Thesis, Department of Botany, University of Dhaka. pp. 129.

25. Akter R 2016. Responses of BARI Sweet Corn-1 (Zea mays L.) to Naphthalene acetic acid at various nitrogen fertilizer levels. M.Sc Thesis, Department of Botany, Jagannath University, Dhaka, Bangladesh. pp. 158. 